\title{
Interoceptive conditioning: An attempt at replication'
}

C. G, COSTELlo AND KEN LOW

THE UNIVERSITY OF CALGARY
An attempt was made to replicate Okhnyanskaya's (1953) experiment in which she conditioned a respiratory vasomotor reflex. The attempt failed due to the extremely variable and unstable nature of the vascular responses consequent upon deep inhalation.

An experiment reported by Okhnyanskaya (1953) was presented by Razran (1961) as ". . . an example of 'natural' exterointeroceptive conditioning." His account is as follows:

"Experimenter noted that when his subjects were told to inhale, they manifested clear-cut plethysmographic arm vasoconstriction in addition to changes in their pneumograms as may be seen in the left portion of Figure 17 [reproduced here as Fig. 1]. The particular vasoconstriction is apparently a viscerovisceral vasomotor reflex evoked by the respiration. Now, the experimenter told his subjects not to inhale when the word 'inhale' was given. But, as may be seen in the right portion of the figure, the vasoconstriction persisted despite the entirely regular pneumogram -it was, presumably, 'naturally" conditioned to the word 'inhale' and was not deconditioned-or inhibited-by the instructions not to inhale when 'inhale' is uttered . . ." (Razran, 1961, p. 92).

The experiment seemed ". . .by all tokens to shed light on two intricate CR mechanisms: (a) the chaining of classical conditioning and the CR efficacy of a three-link exteroceptive/interoceptive CR chain when the middle link is omitted, and (b) the deconditioning or reversing power of instructions upon previously conditioned verbal stimuli. The experiment is obviously one that could be readily duplicated-and expanded-in any American laboratory" (Razran, 1961, p. 92).

Reference to the original article by Okhnyanskaya ${ }^{2}$ suggested that Razran had misinterpreted her paper. Okhnyanskaya does not call the link between the word "inhale"' and the vasoconstriction a "naturally conditioned link." She reports the experimental classical conditioning of the respiratory-vascular reflex to the word "inhale."

Replication was difficult since in the original paper: (1) Subject characteristics were not reported other than the fact that they were suffering from lead and mercury poisoning, which immediately casts doubt on the generality of the findings. (2) No apparatus descriptions were presented. (3) Details of the pro- cedure used (number of trials, intertrial intervals, etc.) were not presented. 3 (3) A chart speed of $25 \mathrm{~mm}$ per sec was reported which, if correct, could not have resulted in the figures presented in the paper. STUDY 1

\section{Subjects}

There were five volunteer $\mathrm{Ss}$, three female and two male, whose ages ranged from 21 to 35 years.

\section{Apparatus and Procedure}

Pneumographic and digital plethysmographic recordings were made on a Grass Model 7 polygraph. Ss reclined in a half sitting position on an adjustable hospital bed in a lighted, soundproofed room. Because the failure to obtain conditioning appeared to be due to the variability of the unconditioned response, the conditioning procedure will not be described.

\section{Resulits}

Digital vasoconstriction did not consistently occur for all Ss on inhalation. There was considerable inter-S variability and intra- and inter-session variability for individual Ss. Conditioning did not occur. It was decided in the second study to use an arm plethysmograph as had been used by Okhnyanskaya.

\section{STUDY 2}

\section{Subjects}

There were nine volunteer Ss; eight female and one male, whose ages ranged from 20 to 24 years.

\section{Apparatus and Procedure}

The recording procedure was the same as that used in Study 1 except that plethysmographic recordings were made by a specially constructed oncometer large enough to allow insertion of the arm past the elbow. Results

The recordings obtained by the method showed far less inter-S variability, and most of them on deep inhalation showed the vasoconstriction pattern reported

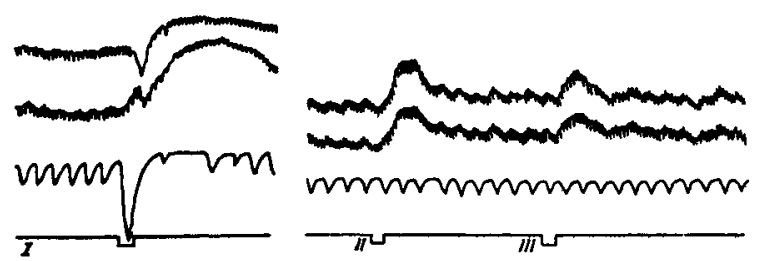

Fig. 1. Conditioned interoceptive vascular changes in human Ss. Plethysmogr ams and pneumograms to the sound of the word "inhale" when (a) the subjects were told to inhale-left portion of figure-and when (b) they were told not to inhale when they heard the word "inhale"-right portion of figure (from Raxan, 1961). 
by Okhnyanskaya. In Fig. 2 a typical recording is presented.

But with this apparatus a large arm movement artifact occurred during deep inhalation. Various positions of the arm and of the plethysmograph on the arm were investigated but the artifact could not be eliminated. No evidence of conditioning, even after 185 trials, was obtained. A final attempt was made at replicating Okhnyanskaya's findings using a digital plethysmograph.

\section{STUDY 3}

\section{Subjects}

There were five female volunteer Ss, whose ages ranged from 21 to 22 years.

Apparatus

The apparatus was the same as that used in Study 1. Results

As in Study 1, considerable variation in the pattern of the vascular response to deep inhalation was obtained.

It would appear that though constriction is usually one part of the total response, it is by no means always the dominant part.

In Fig. 3, three types of patterns are presented to illustrate the variability of response.

A number of other observations were made on this particular unconditioned reflex during the three studies, suggesting that Okhnyanskaya's experiment is by no means ". . obviously one that could be readily replicated. .."

(1) The vascular response grows smaller within sessions and often disappears altogether within five to 10 trials. One immediately suspects that part of the response may be an orienting one to the novelty of deep inhalation (Sokolov, 1963). Whether or not this is so, it is clear that it would not be easy to condition a response that so readily adapts out.

(2) The pattern of response, though it generally remained the same, as long as it lasted within a
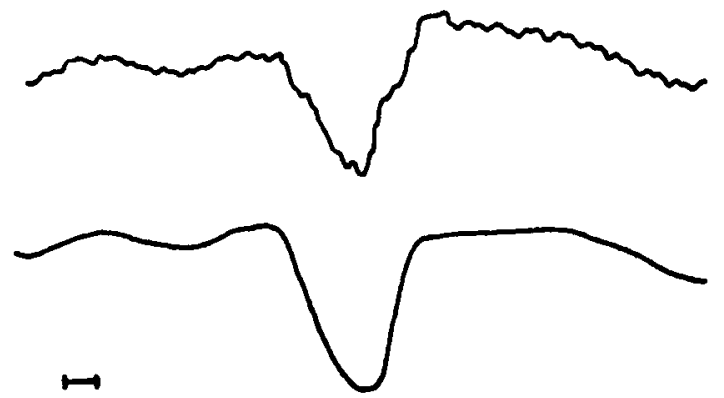

$1 \mathrm{Sec}$

Fig. 2. Plethysmogram (bottom) and pneumogram (top) when the Ss inhaled.
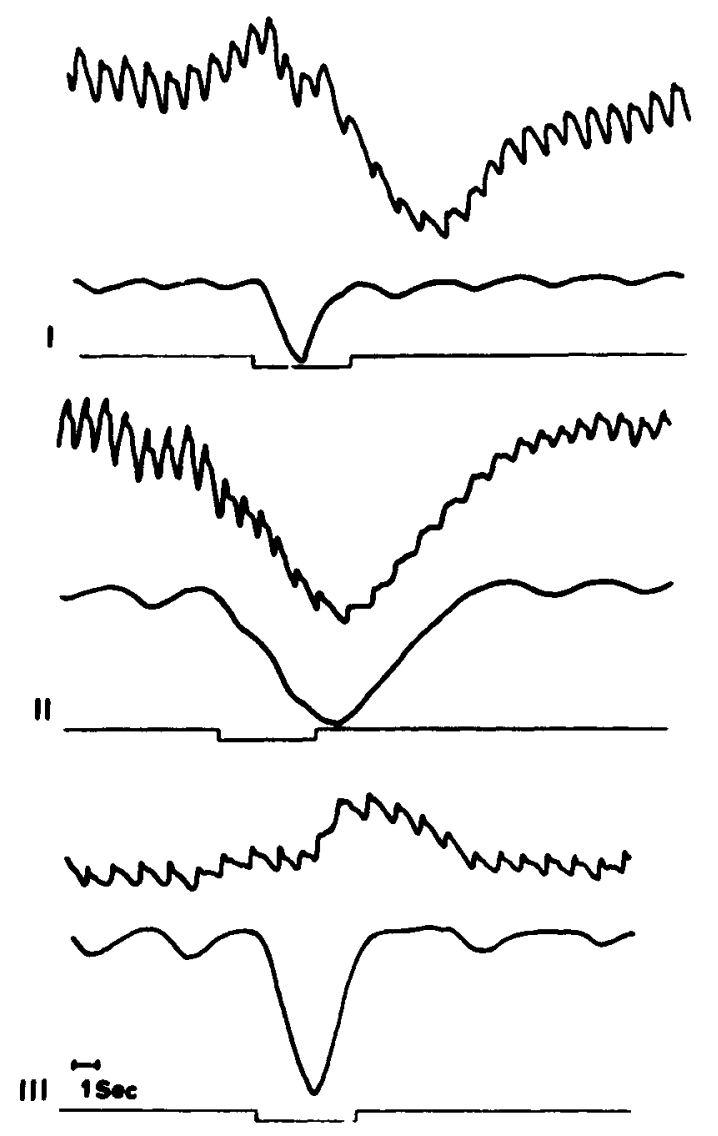

Fig. 3. Three types of vascular responses on inhalation from three different Ss.

session, usually changed considerably from one session to another.

(3) Variability of response was clearly a function of intertrial interval. Consistency in the pattern of responding, even within sessions, was difficult to obtain with intertrial intervals less than $5 \mathrm{~min}$.

\section{References}

Okhnyanskaya, L. G. A study of the conditioned respiratory vasomotor teflexes: Respiration as the stimulus of vasomotion. Fiziol. Zl. SSSR., 1953, 39, 610-613.

Razran, G. The observable unconscious and the inferable conscious in current Soviet psychophysiology: Interoceptive conditioning, and the orienting reflex. Psychol. Rev., 1961, 68, 81-147. Sokolov, Ye. N. Perception and the conditioned reflex. New York: Pergamon Press, 1963.

\section{Notes}

1. Research done under Grant APA-138 from the National Research Council of Canada.

2. Thanks are due to John Adamowicz for his translation of the article.

3. Correspondence with the author of the original article did not shed any further light on these matters. 